\title{
Aparición de delirio de Capgras tras afección por hemorragia subaracnoidea en paciente con trastorno mental grave.
}

Emergence of Capgras delusion after a subarachnoid hemorrhage in a patient with severe mental disorder.

\author{
María Angeles Alayeto Gastón a , Naiara Sánchez Lucas b \\ ${ }^{a}$ Residente de Psicología Clínica.- Hospital General San Jorge, Huesca, España. ${ }^{b}$ Residente de Psicología \\ Clínica.- Hospital General San Jorge, Huesca, España.
}

Correspondencia: María Angeles Alayeto Gastón (ma.alayeto@gmail.com)

Recibido: 31/12/2012; aceptado: 20/05/2013

\begin{abstract}
RESUMEN: Se presenta el caso de una paciente con trastorno mental grave que tras sufrir una hemorragia subaracnoidea comienza a manifestar la idea delirante consistente en que unos dobles usurpan la identidad de personas allegadas a ella (delirio de Capgras). Se observa a la paciente durante proceso rehabilitador en unidad de media estancia dos años después del episodio HSA, donde ingresa por empeoramiento y viraje psicopatológico. Las distintas teorías o modelos que por sí solos han intentado dar cuenta de la etiopatogenia del delirio de Capgras no consiguen explicarlo de forma satisfactoria. Aunque algunos enfatizan su origen orgánico la realidad es que no hay localización de un daño común para todos los casos, y no siempre es posible determinarlo. El interés del caso que aquí se estudia estriba en la coexistencia de diversos factores implicados, orgánicos y no orgánicos, así como en la propia resolución del delirio. Utilizando un enfoque etiopatogénico multifactorial se hipotetizará acerca de la particular formación del delirio de Capgras en la paciente, atendiendo a las propuestas teóricas existentes e introduciendo cuestiones idiosincrásicas pertinentes, factores personales y ambientales que no deben obviarse. Tras el análisis del caso y en base a algunos resultados hallados se discute finalmente acerca de cuestiones tales como la unicidad del fenómeno y sus posibles implicaciones terapéuticas.
\end{abstract}

PALABRAS CLAVE: Capgras. Delirio. Síndrome de Capgras. Etiología. Neuropsicología.
ABSTRACT: It presents a case of a patient with severe mental disorder who after suffering a subarachnoid hemorrhage starts manifiestar the delusion that some doubles are usurping the identity of people close to her (Capgras delusion). It observes the patient during the rehabilitation process in medium-stay unit two years after the episode HSA, where entered for worsening and the psychopathology evolution. The various theories or models alone that have tried to explain the etiology of Capgras delusion fail to explain successfully. Although some emphasize an organic origin, the reality is that no damage on a common location for all cases and not always possible to determine it. The interest of the case studied here lies in the coexistence of different factors involved, organics and not organics, and particular resolution of the delirium. Using a multivariate approach it has hypothesized about the Capgras delusion singular formation in the patient, taking into account the existing theoretical proposals and introducing relevant issues idiosyncratic, personal and environmental factors, that should not be ignored. After analyzing the case and based on some results finally it has found out, it discussed about issues such as the oneness of the phenomenon and its possible therapeutic implications.

KEY WORDS: Capgras. Delusion. Capgras Syndrome. Etiology. Neuropsychology 


\section{Introducción}

El delirio de Capgras (DC) o "ilusión de los dobles", término acuñado por Jean Marie Joseph Capgras y J. Reboul-Lachaux en 1923 (1) supone la creencia de que una persona conocida y afectivamente significativa, es reemplazada por un impostor idéntico a esa persona. El DC puede presentarse de manera crónica o transitoria, en ocasiones fluctuante en el tiempo (2). Más frecuente en mujeres, en una relación de $3: 2$ y no encontrándose diferencias entre edades. Se observa en diversos trastornos mentales graves y se relaciona con múltiples enfermedades somáticas (3). Destacable la alta prevalencia del DC en demencias y otros procesos neurodegenerativos (3-4), estimada en la enfermedad de Alzheimer hasta en casi un tercio, y hasta un 17\% en la demencia por cuerpos de Lewy (5).

Las teorías cognitivas sugieren, en general, que durante el procesamiento de la información se produciría una ruptura entre las tres primeras fases del proceso -codificación, análisis y procesamiento de la información- y la última, - identificación y reconocimiento- (6). Los primeros modelos, como el de reconocimiento facial propuesto por Bruce y Young se basan en que la "sensación afectiva de familiaridad" evocada al percibir caras, emergería por la integración de distintas unidades destinadas al "reconocimiento" de voz, cara, gestos... $(2,3,7)$. Esta integración resultaría fallida en el caso del DC, imposibilitándose la identificación personal. En este sentido Pacherie (8) tomando el concepto de modularidad fodoriana habla de un "dominio específico" encargado del procesamiento afectivo de las caras. Otros autores (9) van un paso más allá, al afirmar que lo queda distorsionada en realidad es la "sensación de unicidad" o "singularidad" (de una persona, animal, objeto o lugar).

Más recientemente y en un intento por conjugar el conocimiento aportado por las teorías cognitivas con los hallazgos neuroanatómicos que las técnicas de neuroimagen revelan, y coincidiendo con el uso de exploraciones neuropsicológicas más exhaustivas, surgen distintos modelos neuropsicológicos. Algunos de estos modelos holísticos postulan la existencia de un fallo al conciliar la información sobre el reconocimiento y las emociones (teorías de desconexión), quedando implicadas algunas estructuras occipito-temporales; otros en cambio se centran en el papel que juega el hemisferio derecho (teorías de lateralización) sustentado en el hecho de que funciones como la automonitorización, la monitorización de la realidad, la memoria y la sensación de familiaridad quedan comprometidas y considerando, además, la necesaria preservación del hemisferio izquierdo para su aparición; y los últimos, que señalan el protagonismo del lóbulo frontal (teorías frontotemporales), observándose distintas combinaciones de daño en los lóbulos frontal (derecho de forma mayoritaria), temporal (incluyendo regiones límbicas) y en ocasiones parietal $(2,3,5,6)$.

Otros factores estudiados partícipes de la etiopatogenia del DC tienen que ver con la personalidad previa, el afecto y aspectos motivacionales, psicodinámicos y 
atribucionales $(2,5,6)$, también con el razonamiento y la presencia de algunos sesgos cognitivos (10-11).

No obstante ninguno de los modelos expuestos por sí solos pueden dar cuenta de la formación del DC. Se desprende la necesidad de manejar hipótesis etiopatogénicas multifactoriales. El análisis de casos clínicos se considera un método muy útil en este sentido, pues permite un acercamiento mucho más ecológico, desde la complejidad y riqueza del ser humano, capaz de revelar claves que estimulen otras investigaciones más ambiciosas; en el caso que presentamos a continuación sin dejar de contemplar y contrastando con los acercamientos teóricos existentes, se atienden a todos aquellos aspectos de la paciente que se considera probablemente estén implicados en la génesis del DC.

\section{Presentación del caso}

Antecedentes y aparición del Delirio de Capgras tras Hemorragia Subaracnoidea (HSA)

Paciente de 59 años, licenciada en ciencias exactas y ex profesora de instituto. Es la mayor de tres hermanos. Describe su infancia feliz, estaba muy unida a su padre, era una niña extrovertida, sociable e independiente. Intelectualmente brillante, pronto vive de forma independiente trasladándose varias veces de ciudad según requerimientos profesionales. Debutó con un brote psicótico con ideación paranoide a los 30 años de edad tras suspenderse su proyecto de boda. Los episodios maniacos y depresivos se van alternando precisando algunos de ellos ingreso hospitalario, aunque pudiendo desarrollar vida normal sin afectación laboral entre periodos hasta el tercero de sus ingresos en el año 2005, motivado por un episodio depresivo y coincidiendo con el reciente fallecimiento del padre, momento en el que la paciente solicita la incapacitación laboral (ILP absoluta concedida en 2006). Las recaídas siempre tuvieron que ver con el abandono del tratamiento con Litio. También se observa un consumo abusivo de alcohol durante este tiempo. El diagnóstico hasta diciembre de 2009: "Trastorno Bipolar con síntomas psicóticos congruentes y no congruentes, con remisión" y "Abuso de alcohol". Desde que se independiza apenas tiene relación con sus padres y hermanos, por su estilo de vida y sintomatología maniaca es considerada como la "oveja negra" de la familia.

En 2009 sufre una Hemorragia Subaracnoidea (HSA). El TAC revela afectación izquierda temporo-parietal que se rodea de amplio edema; efecto masa sobre el sistema ventricular ipsilateral y hematoma parietal izquierdo agudo con afectación grave del nivel de conciencia, sensibilidad, motricidad y habla. Al despertar del coma dice que lo primero que ve es la cara de un antiguo compañero profesor al que le unía 
una relación de afecto. Tras esto habla de que sus hermanos "no son sus hermanos", atribuyendo al profesor amigo el cambio. El DC es extensible a amigos y conocidos. Abandonada por su pareja en ese momento, será el hermano quien se responsabilice de su cuidado.

Evolución del caso tras HSA. Posteriormente requiere varios ingresos hospitalarios por descompensación de síntomas psiquiátricos; prácticamente deja de haber periodos asintomáticos. En unidad de agudos previo al ingreso en UME presentaba: potomanía, descompensación psicótica con ideación delirante tipo paranoide, alucinaciones cenestésicas, aislamiento social y pensamiento desorganizado, ánimo hipertímico, insomnio, y ligera desinhibición. Se retiraron las sales de litio por nefropatía secundaria a diabetes insípida, instaurándose ácido valproico. El diagnóstico: "Trastorno Esquizoafectivo mixto, con síntomas psicóticos congruentes y no congruentes"; "Abuso de alcohol". En febrero de 2012 ingresa en UME para iniciar un "plan individualizado de rehabilitación".

- La evaluación médico-enfermera constató una limitación funcional moderada con hipotonía generalizada. Antecedentes de hipotiroidismo y DM-NID, ambos en remisión. Potomanía y enuresis secundarias a episodios de diabetes insípida nefrogénica que se corrigieron con autoregistro y tiacídicos.

- La exploración neuropsicológica (WAIS IV) evidenció deterioro cognitivo tanto en memoria operativa como en velocidad de procesamiento, aunque los niveles reflejan un nivel "normal", en torno a CI de 100, se presupone con anterioridad mucho más elevada atendiendo a su historia académica y profesional. Exhibe conductas verbales socialmente inapropiadas que sugerirían afectación frontal, quizá consecuente con la dilatada trayectoria de consumo abusivo de alcohol.

- Evaluación psicopatológica: Presencia de varios delirios, de influencia corporal, de telepatía y DC, y algún fenómeno alucinatorio simple. El mismo profesor que provoca suplantación de identidades también le tira cosas y le empuja. Se comunica telepáticamente con este a través de los números que aparecen en la TV y de los chasquidos que escucha en los cristales. Las características del DC se recogen en la tabla 1. Destacar que aunque al principio manifestaba algo de hostilidad hacia el "doble del hermano", los sentimientos pasaron a ser positivos, de hecho ahora se lleva mucho mejor con él que antes del episodio de la HSA. La estabilización psicopatológica se consigue con Depakine 1300 mg/día, Olanzapina 20 mg/día, Risperidona $6 \mathrm{mg} /$ día, Complejo Vitamina B. Participa en el "grupo de adherencia al tratamiento". Alta en diciembre de 2012. Sintomatología psicótica positiva residual e insight parcial. 
Tabla 1.

Manifestación del Delirio de Capgras en la paciente.

\begin{tabular}{|ll|}
\hline Edad de aparición & • Tardía, 55 años \\
\hline Sintomatología psiquiátrica premórbida & • Sintomatología paranoide durante episodios maniaco/depresivos \\
\hline Deterioro cognitivo & • Considerable: Memoria Operativa y Velocidad de Procesamiento \\
\hline Permanencia de la creencia & $\begin{array}{l}\text { - Desde Hemorragia Subaracnoidea (de diciembre de } 2009 \text { hasta } \\
\text { final 2012) }\end{array}$ \\
\hline Inicio del cuadro & • Brusco, tras Hemorragia Subaracnoidea \\
\hline Experiencias anómalas & $\begin{array}{l}\text { - Imagen hipnapómpica/alucinatoria al despertar ("ve" al profesor } \\
\text { colega) } \\
\text { Sensación de extrañeza, desafectación respecto a hermano y co- } \\
\text { nocidos. }\end{array}$ \\
\hline Elaboración del delirio & $\begin{array}{l}\text { - Un profesor colega de profesión al que "ve" tras despertar del } \\
\text { episodio HSA es el que ha cambiado a su hermano y a otros cono- } \\
\text { cidos por otras personas }\end{array}$ \\
\hline Resolución del delirio & $\begin{array}{l}\text { - Atípica por rapidez, ahora la atribución respecto al hermano: } \\
\text { "será el Capgras"; "le habrá cambiado personalidad" } \\
\text { - Coincide con estabilización psicopatológica } \\
\text { - Número reducido de sesiones individuales de terapia cognitivo- } \\
\text { conductual consistente en la búsqueda de explicaciones alternati- } \\
\text { vas y psicoeducación. } \\
\text { • Sesiones grupales "Conciencia de enfermedad" }\end{array}$ \\
\hline Adaptación al doble & \begin{tabular}{l} 
Escasa muestra de rechazo u hostilidad, buena adaptación \\
\hline
\end{tabular} \\
\hline
\end{tabular}

\section{Discusión}

Para facilitar el seguimiento en la tabla 2 se compilan los principales factores asociados al DC.

La deriva psicopatológica hacia sintomatología psicótica crónica tras el episodio de hemorragia subaracnoidea sufrida, apunta a tal suceso como desencadenante de la misma. Es precisamente después de este episodio cuando de forma brusca aparece la idea delirante de suplantación. La literatura señala algunas diferencias en la manifestación del DC según el origen esté asociado a organicidad o a trastorno mental grave (2). Prueba de un origen orgánico del DC en el caso de esta paciente son la aparición tardía y de forma brusca tras la HSA, la constatación por TAC de daño cerebral en zona temporo-parietal (izquierda) y la sospecha de daño frontal leve, el considerable deterioro cognitivo observado, la clínica previa paranoide (presente durante algunos de los episodios maniaco-depresivos), la persistencia del delirio pese a no 
provocarle gran repercusión emocional, la ausencia de agresividad hacia "el doble" y la excelente adaptación al mismo. También el delirio no muy elaborado, aun esto subjetivo de determinar y extraña la explicación que la paciente ofrece en un principio, sí parece diferir en este sentido respecto a las explicaciones delirantes típicas de la esquizofrenia. Los síntomas paranoides concurrentes al DC se relacionan con origen psiquiátrico; si bien cierto que concurren otros delirios la paciente parece vivirlos como una "forma de comunicarse" o como "un juego" entre su amigo y ella. A tenor del análisis de las características presentadas, parece que el daño orgánico tendría gran peso en el desarrollo del Capgras. El impacto neuroanatómico tras la HSA pudo provocar la vivencia de algunas experiencias anómalas, la paciente refiere la visión del amigo profesor al despertar, la extrañeza ante personas cercanas escasamente reconocibles e incluso la torpeza motora que le hace caerse y tirar objetos. Los intentos por dar explicación a estas experiencias inusuales y extrañas, mediado por unos

Tabla 2 .

Factores relacionados con el Delirio de Capgras.

\begin{tabular}{|c|c|}
\hline - Trastornos psiquiátricos $(2,3,7)$ & $\begin{array}{l}\text { Cuadros esquizofrénicos y esquizoafectivos } \\
\text { Trastorno delirante } \\
\text { Episodios maniacos o depresivos con síntomas psicóticos }\end{array}$ \\
\hline - Neurotransmisores (6) & Hiperactividad dopaminérgica \\
\hline - Enfermedades somáticas $(3,5,6)$ & $\begin{array}{l}\text { Metabólicas: déficit vitamina B12 y ácido fólico } \\
\text { Endocrinas: hiporoidismo, diabetes mellitus } \\
\text { Cerebrales: tumores, demencias, traumatismos, encefalitis, epilepsia, ic- } \\
\text { tus, enfermedad de Huntington, esclerosis múltiple... } \\
\text { Arteriosclerosis } \\
\text { Intoxicaciones (litio, cloroquina...) } \\
\text { Enfermedades postparto }\end{array}$ \\
\hline - Alteraciones orgánicas (2-5) & $\begin{array}{l}\text { Más frecuente afectación del hemisferio derecho } \\
\text { Afectación frontotemporal y parietal } \\
\text { Afectación lóbulos frontales bilaterales } \\
\text { "Desconexión" vía dorsal (occipito-temporal), implicación amígdala } \\
\text { Más frecuente hemisferio izquierdo preservado... }\end{array}$ \\
\hline - Personalidad previa (2) & Paranoide \\
\hline $\begin{array}{l}\text { - Aspectos psicodinámicos y moti- } \\
\text { vacionales }(2,5,6)\end{array}$ & $\begin{array}{l}\text { Conflictos afectivos, pulsiones; Fragmentación del yo; Conservación de } \\
\text { la autoestima; Defensas: proyección, identificación proyectiva, regresión, } \\
\text { escisión... }\end{array}$ \\
\hline - Estilo atribucional $(2,6)$ & Externalizante, atribuir lo que ocurre a "los otros" \\
\hline - Estado de ánimo $(2,6)$ & Suspicacia, incertidumbre \\
\hline - Razonamiento $(12,14)$ & $\begin{array}{l}\text { Saltos bruscos de los datos observados a las conclusiones } \\
\text { Errores razonamiento (silogístico, probabilístico) }\end{array}$ \\
\hline $\begin{array}{l}\text { - Aspectos cognitivos } \\
(2,5,6,7,9,10,12)\end{array}$ & $\begin{array}{l}\text { Fallo emergencia sensación de familiaridad } \\
\text { Fallo emergencia sensación de unicidad } \\
\text { Sesgo cognitivo: correlación ilusoria } \\
\text { Fallo cognición social (intencionalidad de los otros) }\end{array}$ \\
\hline
\end{tabular}


niveles dopaminérgicos probablemente alterados en correspondencia a la patología psiquiátrica de base, se hipotetiza tuvieron que ver con la presentación delirante final. Congruente con la "teoría perceptiva" de Maher (10), quien situaría el inicio de cualquier delirio en un problema perceptivo de origen biológico que convirtiéndose en un suceso extraño, incomprensible para el sujeto, este trataría de interpretar y dar algún sentido. Además la creencia delirante se mantendría por refuerzo negativo, al quedar aliviada la incertidumbre y la tensión al poder justificar tal experiencia anormal (11). Nótese como pudo producirse un sesgo cognitivo, una "correlación ilusoria" (11) entre dos eventos que se suceden en el tiempo, como fueron la visión del amigo y la extrañeza al no reconocer a su familia, la primera confirmándose como causa de la segunda, responsabilizando a su amigo de la usurpación de los cuerpos. Según y conforme se va produciendo la estabilización psicopatológica por el mantenimiento del tratamiento antipsicótico pautado la paciente será capaz de aprovechar la terapia psicológica logrando elaborar algunas explicaciones alternativas no delirantes. Ahora la evidencia de que el hermano es más amable, cuidador, y se preocupa por ella es justificada a través de un cambio de personalidad o incluso por la manifestación de un DC.

Aspectos afectivos y motivacionales parecen relevantes: El fallecimiento del padre, el abandono por su pareja y el distanciamiento de su familia durante años. No parece casual el peso que ahora otorga a dos figuras masculinas, impresiona que aunque "reales" una aparezca desconocida para ella, su hermano y con la otra mantenga una relación sólo en su mente, el amigo profesor. Posiblemente en muchos momentos la paciente pudo haber percibido desaprobación por parte de los miembros de su familia, ocasionada por la improcedencia y molestia que algunas de sus actuaciones en el pasado hubieran podido causarles, sobre todo las relacionadas con sus episodios maniacos o con el abuso de alcohol. Esto pudo hacerle albergar sentimientos ambivalentes al respecto, deseo de afecto y compañía en un momento de gran vulnerabilidad vital aunque cierto rechazo inconsciente a la idea de que un miembro de su familia sea precisamente el que lo satisfaga.

Destacar que una de las explicaciones alternativas que ahora adjudica al DC en relación a su hermano, el cambio de personalidad de este, comporta dos cuestiones muy relevantes, la primera que debió producirse una pérdida de reconocimiento afectivo tras la HSA, y la segunda es que a día de hoy no ha debido de recuperarla, puesto que no deja de ser una respuesta ante la extrañeza o a la ausencia de "sensación de familiaridad" percibidas. Por otro lado respecto a la segunda de las atribuciones que hace, al padecimiento de un síndrome psiquiátrico, si bien le otorgaría cierto grado de conciencia de enfermedad, tampoco hace pensar en la recuperación de tal reconocimiento emocional. Lo que sin duda recupera es la capacidad para realizar una atribución no delirante. En este caso en concreto, el daño orgánico obraría como condición necesaria aunque no suficiente para la génesis del DC, afectando al proceso 
de reconocimiento e identificación, pero emergiendo la interpretación delirante por la conjunción de otros factores analizados. Para poder certificar el fallo en el "reconocimiento emocional" hubiera sido deseable haber podido realizar mediciones de la respuesta autonómica de conducción de la piel, al modo de las que se efectúan desde los modelos neuropsicológicos "de desconexión" (2).

Parece entonces que por lo menos en algunos casos la expresión delirante del DC se conformaría tanto por una "falsa identificación", en ocasiones pudiendo desencadenarse por alteraciones cerebrales (ya sean estas temporales o permanentes), como por la formación de atribuciones o creencias delirantes al respecto. Esta confluencia de procesos podría justificar también algunos fenómenos patológicos en los que se observan alteraciones en la identificación por errores en la conciliación "reconocimiento cognitivo" y "sensación de familiaridad", tales como los que junto al DC son denominados "síndromes de falsa identificación delirante" (12) (ver figura 1) y la prosopagnosia $(4,12)$, e incluso otros bastantes comunes en la población general, como la desrealización, la despersonalización $(3,5,6)$ y las paramnesias temporales "déjà vu" y "jamais vu"(4).

Para estas y otras manifestaciones en las que se producen "errores de identificación" y en la línea de otros autores (5) aquí se propone una conceptualización dimensional, atendiendo a: reconocimiento afectivo (sobreidentificación versus ausencia de identificación) reconocimiento cognitivo (presente versus ausente) y atribución (delirante versus no delirante). Únicamente en los "síndromes de falsa identificación delirante" se darían atribuciones delirantes.

Si se admite esta doble vía "fallo de identificación-atribución", dirimir entre origen orgánico o funcional del DC, supondría en realidad hacerlo respecto al propio origen del "error de identificación", cuya relevancia por otro lado tal vez resida en la oportunidad de que este fallo pudiese revertirse.

Figura 1.

Síndromes de falsa identificación.

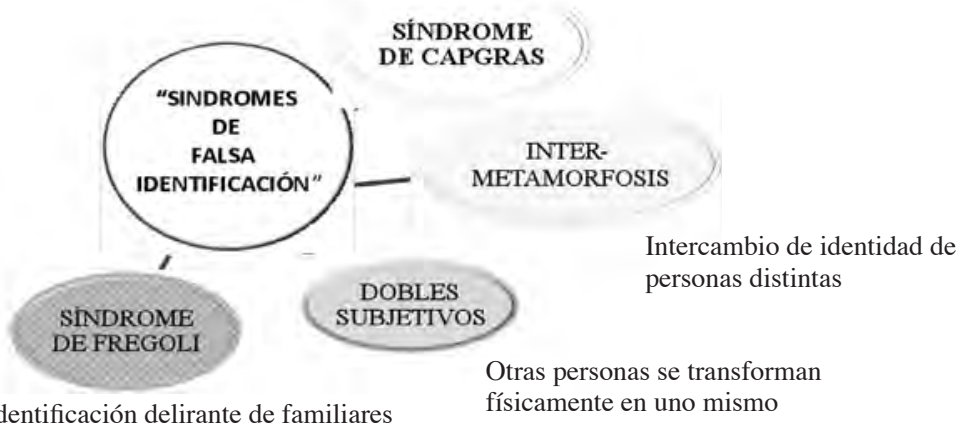

Sobreidentificación delirante de familiares físicamente en uno mismo en el cuerpo de otras personas extrañas 
Para concluir, las investigaciones recientes apoyan el uso de terapia cognitivo conductual para potenciar la acción de los fármacos antipsicóticos en el tratamiento de síntomas delirantes (13), ayudando al paciente a desconfirmar creencias o a sustituirlas por otras; por lo que en aquellos casos en los que aceptemos una disociación en el fenómeno de Capgras, aplicar técnicas cognitivo-conductuales sería indispensable, pues permitiría ayudar al paciente a moldear y favorecer explicaciones más adaptativas y menos angustiosas, y posiblemente reducir la frecuencia de las conductas agresivas que muchas veces presentan hacia "el impostor" o "impostores".

\section{BIBLIOGRAFÍA:}

(1) Capgras J, Reboul-Lachaux J. L'illusion des sosies dans un dèlire systématisé chronique. Bull Soc Clin Méd Ment 1923; 2:6-16.

(2) Madoz-Gúrpide A, Hillers- Rodríguez R. Delirio de Capgras: una revisión de las teorías etiológicas. Rev Neurol 2010; 50: 420-30.

(3) Hillers-Rodríguez R, Madoz-Gúrpide A, Tirapu J. Propuesta de una batería neuropsicológica para la exploración del síndrome de Capgras. Rev Esp Geriatr Gerontol 2011; 46(5):275-280.

(4) Moreno A. Las falsas ideaciones en demencia: contenido y características de los fenómenos delusionales. Alcmeon. Revista Argentina de Clínica Neuropsiquiátrica [revista electrónica] 2009 julio. [acceso el 10 de diciembre de 2012]; 15 (60): 288-295. Disponible en: http://www.alcmeon.com. ar/15/60/04_toledo\%20alcmeon60.pdf

(5) Morrillo-Velarde Q, López AI, Santamaría L. Síndrome de Capgras: Análisis crítico a propósito de dos casos. Psiq Pública 1998; 10: 233-238.

(6) Manzano JM, Muñoz JJ. Delirio de Capgras: Una revisión. Psiquiatria.com [revista en Internet] 2011 noviembre. [acceso el 1 de diciembre de 2012]; 15:62. Disponible en: http:/hdl.handle. net/10401/4848

(7) Gallego L, Vázquez S, Peláez JC y López-Ibor JJ. Aspectos neuropsicológicos, clínicos y sociales de dos casos de síndrome de Capgras. Actas Esp Psiquiatr 2011;39 (6):408-14.

(8) Pacherie E. Perception, emotions and delusions: revisiting the capgras' delusion. En Bayne T, Fernandez J, editors. Delusion and Self-Deception: Affective and Motivational Influences on Belief Formation. New York, NY: Psychology Press, 2008; p. 114-115.

(9) Margariti MM, Kontaxakis VP. Approaching delusional misidentification syndromes as a disorder of the sense of uniqueness. Psychopathology 2006; 39: 261-8. 20.

(10) Maher BA. Anomalous Experience and Delusional Thinking: The logic of explanations. En Oltmans TF y Maher BA (eds.). Delusional Beliefs, pp. 15-33. New York, John Wiley \& Sons, 1988.

(11) Fuentenebro F, Valiente C, Diez-Alegría C, Nieto M. Psicopatología de la psicosis: Delirio. Dialnet Informaciones Psiquiátricas 2007; 189.

(12) Montesinos JE, Salas JJ, Sánchez ML y Hernández ML. Síndromes de falsa identificación delirante. Síndrome de Capgras. Presentación de un caso. Alcmeon. Revista Argentina de Clínica Neuropsiquiátrica [revista en internet] 2008 septiembre.[acceso 20 de diciembre de 2012]; 14 (57):24-32. Disponible en www.alcmeon.com.ar/15/57/05_montesinos.pdf 
(13) Perona-Garcelá S, Cuevas-Yust C y Martínez-López M J. Guía de tratamientos eficaces en delirios y alucinaciones. En Pérez M, Fernández JR, Fernández C, y Amigo I, editors. Guía de tratamientos psicológicos eficaces. Vol. I, Adultos. Madrid: Pirámide, 2003.

(14) Olivos P. La mente delirante. Psicopatología del delirio Delusional mind. Psychopathology of delusion. Rev Chil Neuro-Psiquiat 2009; 47 (1): 67-85. 